Check for updates

Cite this: Nanoscale Adv., 2019, 1, 4790

\title{
Environmental fate descriptors for glycol-coated selenium nanoparticles: a quantitative multi-assay approach $\dagger$
}

\begin{abstract}
Savita Chaudhary, ${ }^{\text {aa }}$ Pooja Chauhan ${ }^{a}$ and Rajeev Kumar ${ }^{b}$
The application of selenium nanoparticles (Se-NPs) in the fields of biological and physical science is escalating very fast. Therefore, it is necessary to investigate the effects of Se-NPs for their prospective vulnerability in the environment. The present work involved the investigation of the toxicological responses of four different types of glycols, i.e., EG, DEG, TEG and PEG coated on Se-NPs by using a multi-assay approach. The influence of the surface-coated Se-NPs was examined over four different types of bacterial strains. Additional work was carried out with glycol-coated Se-NPs for observing their anti-oxidative activities. To achieve the objective of assessing the environmental fate of the chosen SeNPs, they were tested for assessing their hydrogen peroxide free radical scavenging activity and total reduction behaviour. The in vitro stability of the surface-coated Se-NPs was investigated over various components of blood. It was observed from the data that $15 \%$ glycol-encapsulated Se-NPs were biocompatible with the chosen components. The sorption, degradation and aggregation parameters were investigated to describe the environmental fate of the surface-coated Se-NPs. The results from the multi-assay activities are meaningful for improving the perception about the ecological fate of Se-NPs with their probable threats and reducing the uncertainty about their use.
\end{abstract}

Received 15th October 2019

Accepted 22nd October 2019

DOI: 10.1039/c9na00653b

rsc.li/nanoscale-advances toxicity evaluation of Se-NPs towards the biotic and abiotic species in the environment.

The central theme of this work is to study the effect of surface functionalization on the bio-availability, antimicrobial and antioxidative behaviours of Se-NPs. Se-NPs were also tested for assessing their hydrogen peroxide free radical scavenging activity and total reduction behaviour. The in vitro stability of the surface-coated Se-NPs was studied using various components of blood. The dissolution, aggregation and sedimentation abilities of the surface-coated Se-NPs were also investigated for their safe and secure use.

The present study focused on the synthesis of four different types of Se-NPs using biocompatible coatings of ethylene (EG), diethylene (DEG), triethylene (TEG) and polyethylene glycol (PEG). The surface functionalization of Se-NPs with these four different glycols provided better control over the physiological and biological activities of Se-NPs. ${ }^{17,18}$ The choice of these glycols was made over other templating agents due to their appropriate stabilizing effects and augmentation of the physicochemical properties of Se-NPs by preventing their agglomeration. The glycol molecules completely adhered on the entire surface of the Se-NPs, making them highly biocompatible towards nature. To date, various illustrations have been reported the use of Se-NPs in chemoprevention. ${ }^{19}$ However, the corresponding effect of the colloidal stability of Se-NPs in reaction systems having different $\mathrm{pH}$ values and their sorption, degradation and aggregation parameters have not been 
fully explored simultaneously in any article. The surface coating ability of the chosen glycols made them potential templates for the size-controlled synthesis of Se-NPs. The presence of hydroxyl groups on the surface of the glycol moieties enhanced the surface adsorption ability of Se-NPs and controlled their size. ${ }^{20}$ Furthermore, surface modification with these glycols reduced the chances of non-radiative loss from the prepared nanoparticles and affected their optical and fluorescence properties. The higher water solubility and biocompatibility of the formed nanoparticles enhanced the environmental fate of Se-NPs. The antimicrobial activities of the synthesized nanoparticles were examined using different bacterial strains, i.e., E. coli, S. aureus, S. typhi and $P$. aeruginosa. Also, the effect of the nanoparticles on different components of blood was checked utilizing an in vitro stability assay. Their antioxidant performance was evaluated to diversify their oxidation and free radical species production. The hydrogen peroxide radical scavenging activity and total reduction behaviour of the synthesized nanoparticles were also calculated before their disintegration in the environment. Their aggregation, dissolution, sorption and photodegradation behaviour were also monitored. The detailed profiling of Se-NPs was assessed to measure their level of toxicity and competence for their safe use. The outcomes of this work are useful to specify the dose-responsive and surface coating-dependent relationships of the studied Se-NPs. These investigational results from multi-assay activities are meaningful for improving the perception about the ecological fate of Se-NPs with their probable threats and reducing the uncertainty about their use.

\section{Experimental section}

\section{Materials}

Selenous acid was procured from Himedia with 99\% purity. Ethylene glycol (EG 99.9\%), diethylene glycol (DEG 99\%), triethylene glycol (TEG 99.4\%) and polyethylene glycol (PEG 99\%) were acquired from Sigma Aldrich, Bio Ultra. $\mathrm{NaBH}_{4}$ was used as a reducing agent and purchased from Sigma Aldrich with 98\% purity. Bovine serum albumin (BSA) and human serum albumin were procured in lyophilized forms from Sigma Aldrich with 96\% purity. Dulbecco's modified Eagle's medium (DMEM) was procured from Sigma Aldrich with 98\% purity. L-Histidine and L-cysteine were procured from Himedia in lyophilized forms with $99 \%$ purity. Sodium chloride $(\mathrm{NaCl})$ and potassium ferricyanide $\left(\mathrm{K}_{3}\left[\mathrm{Fe}(\mathrm{CN})_{6}\right]\right)$ were purchased from Fisher Scientific with $98 \%$ purity. Phosphate buffer with $\mathrm{pH}$ of 5.6, 6.4, 7 and 7.4 was prepared by mixing sodium phosphate dibasic and sodium phosphate monobasic. Hydrogen peroxide was obtained from Sigma Aldrich with $30 \%(\mathrm{w} / \mathrm{w})$ ratio. Ammonium molybdate and sodium sulphate were purchased from Himedia with 99\% purity. Ascorbic acid and trichloroacetic acid were purchased from Sigma Aldrich with 99\% purity. All the reagents mentioned above were used as received without any further purification. Deionised water was used for the preparation of all solutions. The bacterial strains of Escherichia coli, Pseudomonas aeruginosa, Salmonella typhi and Staphylococcus aureus were obtained from the Department of Microbiology, Panjab University, and Chandigarh, India.

\section{Synthesis of glycol-coated selenium nanoparticles}

For the synthesis of the glycol-coated Se-NPs, three different weight percentages of the four chosen glycols, i.e., $5 \%, 10 \%$ and $15 \%$ were added to deionised water. Then, $0.03 \mathrm{M}$ solution of selenous acid was subsequently added to the respective glycol solution under stirring. This suspension was kept at stirring for the formation of a homogenous solution in an aqueous medium. Also, $0.1 \% \mathrm{NaBH}_{4}$ solution was added to the above prepared solution under stirring. The as-formed solution was again kept for stirring for $5 \mathrm{~h}$ until the colour of the dispersing solution changed from light-yellow to bright-orange. The orange shade of the formed solution confirmed the formation of Se-NPs. The obtained particles were centrifuged at $5000 \mathrm{rpm}$ to remove the larger aggregates. The resultant particles were further washed with water and ethanol.

\section{Instrumentation}

A LABINDIA UV-vis spectrophotometer was used to measure the optical absorption properties of the glycol-coated Se-NPs in the wavelength range of $200-800 \mathrm{~nm}$. The emission properties were examined using a PerkinElmer LS 55 PL spectrophotometer. A dynamic light scattering instrument from Malvern (ZEN 1690) was used to scrutinize the size and polydispersity index of the glycol-coated Se-NPs. The interaction and capping ability of the glycols on the surface of Se were assessed via Fourier transform infrared (FT-IR) spectroscopy (PerkinElmer (RX1)) in the wavenumber range of $4000-400 \mathrm{~cm}^{-1}$. Thermogravimetric analysis (TG-DTA) with an SDT Q-600 instrument was carried out to explore the thermal behaviour of the glycol-coated Se-NPs. DSC measurements (Mettler Toledo (Q20)) with a refrigerated cooling scheme were carried out to inspect the heat capacities and phase transition temperatures of the glycol-coated Se-NPs. Nitrogen gas was utilized as the purge gas with a flow rate of $50 \mathrm{~mL} \mathrm{~min}^{-1}$. The crystalline phases and size of the glycolcoated Se-NPs were assessed using an XRD powder diffractometer (Panalytical X'Pert Pro) with a scan rate of $2 \mathrm{deg} \mathrm{min}^{-1}$ and monochromatic $\mathrm{CuK} \alpha$ radiation in the $2 \theta$ range of $5-90^{\circ}$. Ultracentrifugation of the synthesized nanoparticles was carried using a Remi R-24 centrifuge. A Mettler Toledo digital $\mathrm{pH}$ meter was used to assess the influence of acidic and basic media. HRTEM analysis was performed on JEOL 2100F instrument at $200 \mathrm{kV}$ to investigate the topological alteration of different glycol-coated Se-NPs. EDX analysis was carried out to investigate the purity of the developed nanoparticles utilizing a JEOL JSM 6100 instrument and aqueous solutions of the respective nanoparticles were utilized. The material structure and crystalline quality of the developed glycol-coated Se-NPs were investigated through sophisticated Raman spectroscopy. Raman spectra were recorded in the range of $0-10000 \mathrm{~cm}^{-1}$ by utilizing a LabRAM HR Evolution sophisticated Raman microscope from Horbia equipped with a multilaser and multidetector (excitation wavelength $=750$ and power $=10 \mathrm{~mW}$ ). 


\section{Antimicrobial activities of glycol-coated Se-NPs}

Minimum zone of inhibition assay (MZIs). The in vitro antimicrobial activities of the glycol-coated Se-NPs were determined using the minimum zone of inhibition assay against four pathogenic microorganisms. Briefly, a nutrient broth was used for the growth of E. coli, S. typhi, P. aeruginosa (Gram negative) and $S$. aureus (Gram positive) bacteria. Also, $50 \mu \mathrm{l} \mathrm{ml} \mathrm{m}^{-1}$ concentration of bacteria and glycol-coated Se-NPs were taken during analysis. Deionised water was utilized as a negative control.

Agar well diffusion assay. For different analyses, the agar well diffusion scheme with the minimum inhibitory concentration was adopted. The bacteria cultures were freshly cultured to ensure their activity in 5\%, 10\% and 15\% EG-, DEG-, TEGand PEG-coated Se-NPs. Muller Hinton agar was used for the grounding of plates. For maintaining sterile conditions, the agar solution was autoclaved for $2 \mathrm{~h}$. Then, the agar solution was allowed to stand at room temperature and then poured to set up the agar plates. These plates were incubated at $37{ }^{\circ} \mathrm{C}$ in laminar air flow for $24 \mathrm{~h}$. Subsequently, the bacterial strains were applied to the agar gel in laminar air flow. To investigate the effect of the nanoparticles on the bacterial strains, holes were made with the help of a punch having a diameter of 5 $\mathrm{mm} \cdot{ }^{21-23}$ For this, freshly prepared $10 \mu \mathrm{L}$ suspensions of glycolcoated Se-NPs were used and the respective plates were again incubated for $24 \mathrm{~h}$ at $37{ }^{\circ} \mathrm{C}$. The decrease in the bacteria population $(P \%)$ was calculated using the following formula:

$$
P \%=\frac{A-B}{A} \times 100
$$

here, $A$ and $B$ correspond to the population of bacteria in the control sample and in the presence of the glycol-coated Se-NPs.

\section{In vitro stability assay for glycol-coated Se-NPs}

Before utilizing the synthesized particles for in vivo applications, it is mandatory to scrutinize their in vitro activities for ethical reasons. The respective studies were miniaturised and automated, which led to the screening examination. ${ }^{24,25}$ As one of the major constituents of living beings (primarily human and animals), blood is considered as a primary standard for studying the effect of synthesized nanoparticles. The diverse range of components in blood makes it effective for investigating the in vitro behaviour of the chosen nanoparticles. The stability of the glycol-coated Se-NPs was determined by exposing them in an environment of various blood components including 2\% BSA, $0.2 \mathrm{M}$ histidine, $0.2 \mathrm{M}$ cysteine, $0.9 \%$ saline solution and phosphate buffers having $\mathrm{pH}$ 5.6, 6.4, 7 and 7.5 in the current work. For the analysis, 15\% of EG-, DEG-, TEG- and PEG-coated Se-NPs were utilized. Water was chosen as the reference solvent. For the measurement, the equivalent quantity of glycol-coated Se-NPs was mixed with different types of blood components, followed by incubation at $37{ }^{\circ} \mathrm{C}$ for 2 days. Simultaneously, the stability of $15 \%$ glycol-capped Se-NPs was also investigated in the presence of human serum albumin (HSA) and Dulbecco's modified Eagle's medium (DMEM) after incubation for 7 days at room temperature under optimal conditions. After incubation, the suspension of each sample was collected and allowed to stand at room temperature for 10 minutes. The variations in the absorption capacity of the synthesized Se-NPs in the presence of blood components were investigated using UV-vis spectroscopic analysis and their size distribution profiles were explored through DLS measurements.

\section{Antioxidant assay for glycol-coated Se-NPs}

Total antioxidant activities of glycol-coated Se-NPs. The total antioxidant activities were investigated to ensure the oxidation inhibiting behaviour of the synthesized Se-NPs, which provided a detailed insight into the better understanding of the biocompatibility behaviour of the synthesized nanoparticles. The respective studies involved the analysis of the free radical species produced in the life cycle. The produced free radicals lead to the rupturing of the cells present in different organisms, which decreases their life span. Therefore, before any environmental exposure to the synthesized particles, it is obligatory to ensure their antioxidant behaviour. ${ }^{26,27}$ For the analysis, ascorbic acid was used as a positive antioxidant control. Also, 0.6 M $\mathrm{H}_{2} \mathrm{SO}_{4}, 28 \mathrm{mM}$ sodium sulphate and $4 \mathrm{mM}$ ammonium molybdate solutions were prepared and referred as the TAC reagent. Next, 5\%, 10\% and 15\% EG-, DEG-, TEG- and PEGcoated Se-NPs were prepared in methanol solutions and mixed with above prepared TAC reagent. The prepared samples were incubated at $95{ }^{\circ} \mathrm{C}$ for 1 hour and 30 minutes. A blank standard sample was also prepared in water. UV-vis spectroscopic studies were performed to check the absorbance of the prepared samples at $680 \mathrm{~nm}$. The respective absorbance values were used for calculating the antioxidant percentage of the prepared Se-NPs.

Total reducing power activity of glycol-coated Se-NPs. The corresponding reducing potential of the glycol-coated Se-NPs was investigated for the better understanding of the electron donor and acceptor abilities of Se-NPs. For the measurements, three different concentrations (5\%, 10\% and 15\%) of EG-, DEG-, TEG- and PEG-coated Se-NPs were chosen. ${ }^{28,29}$ For the analysis, $2.5 \mathrm{~mL}$ of the respective solutions of $0.2 \mathrm{M}$ potassium ferricyanide and $1 \%$ phosphate buffer solution $(\mathrm{pH}=6.6)$ were mixed with different concentrations of glycol-encapsulated Se-NPs. The formed solutions were incubated at $50{ }^{\circ} \mathrm{C}$ for 30 minutes. Then, $10 \%$ of trichloroacetic acid solution was added to the above prepared suspensions of Se-NPs. The respective solution was centrifuged at $2000 \mathrm{rpm}$ for 5 minutes. The supernatant was collected and mixed with an equal amount of deionised water. Also, $0.5 \mathrm{~mL}$ of $0.1 \%$ of ferric chloride solution was mixed and stirred well with the supernatant. After stirring for 10 minutes, all the solutions were allowed to stand at room temperature for 5 minutes and their absorbance was recorded at $700 \mathrm{~nm}$ by using a UV-vis spectrophotometer.

Hydrogen peroxide free radical scavenging activity. The hydrogen peroxide free radical scavenging activities of the glycol-coated Se-NPs were further checked as a function of three concentrations of glycol coating (5\%,10\% and 15\%). For the analysis, $10 \mathrm{mM}$ solution of hydrogen peroxide was prepared in phosphate buffer solution (PBS) having $\mathrm{pH}=7.4$. Then, $1 \mathrm{~mL}$ of 
different glycol-coated Se-NPs was mixed with $2 \mathrm{~mL} \mathrm{H}_{2} \mathrm{O}_{2}$ solution in PBS. A blank standard sample was prepared without adding the $\mathrm{H}_{2} \mathrm{O}_{2}$ solution. ${ }^{30,31}$ The absorbance of the respective samples was measured using a UV-vis spectrophotometer. The percentage of $\mathrm{H}_{2} \mathrm{O}_{2}$ scavenging was calculated using the following formula:

$$
\text { Percentage scavenging }=\frac{A_{\mathrm{C}}-A_{\mathrm{S}}}{A_{\mathrm{C}}} \times 100
$$

here, $A_{\mathrm{C}}$ and $A_{\mathrm{S}}$ correspond to the optical density values of the control and nanoparticle sample.

\section{Aggregation and dissolution behaviour of glycol-coated Se- NPs}

Before releasing the synthesized glycol-coated Se-NPs into the environment, it is significant to investigate their aggregation and dissolution behaviours. The aggregation of nanoparticles is advantageous in the context of their environmental fate and decreases their toxicity at the ground level. The dissolution process involves the splitting of the particles and molecules in the subsequent ionic forms, which imparts toxicity. ${ }^{27,32}$ For analysis, $\mathrm{pH}$ ranging from 2 to 12 (acidic and basic media) was selected to study the effect of aggregation. Ten $\mathrm{mL}$ suspensions of $15 \%$ different types of glycol-coated Se-NPs were prepared in different $\mathrm{pH}$ ranges $(2,4,6,8,10$ and 12). On the other hand, $10 \mathrm{~mL}$ suspensions of $15 \%$ different types of glycol-coated SeNPs were prepared in $\mathrm{NaCl}$ solutions with the concentrations of 0,250 and $500 \mathrm{mM}$ to scrutinize the effect of the dissolution of the as-prepared Se-NPs. Different ionic strengths of solutions provided a better opportunity to comprehend the phenomenon of the mobility of ions in the prepared nanoparticles. DLS analysis was carried out to investigate the corresponding changes in the sizes of the Se-NPs as a function of different ranges of $\mathrm{pH}$ and concentrations of $\mathrm{NaCl}$.

\section{Sorption behaviour of glycol-coated Se-NPs}

The sorption behaviour of the Se-NPs was also checked to investigate their environmental fate. For the analysis, dried soil was collected from the Botanical garden, Panjab University Chandigarh. The soil was dehydrated under sunlight for 2 days to prevent bacterial contamination. Then, the obtained soil was sieved (mesh size $0.354 \mathrm{mM}$ ) to remove large-sized particles. To sustain the sterilized condition, the soil was autoclaved for 120 minutes and stored at $-2{ }^{\circ} \mathrm{C}$ for further analysis. The $\mathrm{pH}$ of the obtained soil was $\sim 6.8$ with electrical conductivity of $17.5 \mu \mathrm{S}$ $\mathrm{m}^{-1}$. In addition, the soil possessed $60.04 \%$ clay, $25.06 \%$ sand, $13 \%$ silt and $1 \%$ organic material. ${ }^{33,34}$ For the analysis of the sorption behaviour of the Se-NPs, $10 \mathrm{~mL}$ suspensions of $5 \%$, $10 \%$ and 15\% EG-, DEG-, TEG- and PEG-coated Se-NPs were prepared. Then, $3 \mathrm{~g}$ of autoclaved soil was added to above prepared samples and allowed to stir at $800 \mathrm{rpm}$ for $3 \mathrm{~h}$. Stirring was effective for the adsorption of Se-NPs on the surface of the soil. The as-prepared samples were incubated at room temperature for five days under the influence of normal light. After five days, the supernatants of the respective samples were collected, filtered and analyzed using UV-vis spectroscopy to appraise their optical density values. A control system was set up in deionised water. The optical density values were utilized to calculate the percentage of sorption for the synthesized nanoparticles on the surface of the soil and in the supernatant.

\section{Photo-degradation activity of glycol-coated Se-NPs}

To investigate the photo-degradation propensity of the assynthesized Se-NPs, a Philips lamp having a CFL optical daylight filter along with a $9 \mathrm{~W}$ CFL bulb was used. The respective experiment was carried out at $25^{\circ} \mathrm{C}$. Freshly prepared 5\%, $10 \%$ and 15\% EG-, DEG-, TEG- and PEG-coated Se-NPs were chosen for the analysis. Briefly, $10 \mathrm{~mL}$ solution of each type of Se-NPs was placed in glass vials with tight covering to avoid the chances of evaporation. Subsequently, the respective samples were placed in a chamber under direct exposure of light for 48 hours and incubated at room temperature. One control system was also prepared in distilled water, wrapped with a foil paper and placed with the other vials to contrast the degradation tendency of the nanoparticles. After 48 hours of continuous exposure, UV-vis spectroscopic measurements were recorded to ensure the effect of light by considering the absorbance of the nanoparticles at a specific wavelength. Then, these samples were again placed for 48 hours in the same chamber to estimate their degradation ability. UV-vis spectroscopic measurements were again recorded for the respective samples. The degradation ability was checked using eqn (3) after 48 hours and 96 hours. ${ }^{33,35}$

$$
\text { Degradation }(\%)=\frac{A_{\mathrm{C}}-A_{\mathrm{N}}}{A_{\mathrm{C}}} \times 100
$$

Here, $A_{\mathrm{C}}$ and $A_{\mathrm{N}}$ correspond to the absorbance values of the freshly prepared nanoparticles and after light exposure. The corresponding adsorption efficiency of the chosen nanoparticles was calculated by using the following equation:

$$
\text { Adsorption efficiency }(\%)=\frac{C_{\mathrm{a}}-C_{\mathrm{b}}}{C_{\mathrm{a}}} \times 100
$$

here, $C_{\mathrm{a}}$ and $C_{\mathrm{b}}$ refer to the initial and final concentrations of the nanoparticles before and after adsorption.

\section{Results and discussion}

\section{Structural characterization of glycol-coated Se-NPs}

The structural and optical properties of Se-NPs were investigated to determine the effect of different chain lengths of glycol on the size and optical properties of Se-NPs. The formation of Se-NPs in each type of the chosen glycol was confirmed by the orange coloration of the sample with an absorption hump in the region of 250-350 $\mathrm{nm}$ (Fig. 1). The absence of black coloration further suggested the controlled growth of Se-NPs and better ability of the glycols to prevent the agglomeration of the formed nanoparticles by providing an effective coating over the surface of Se-NPs. In addition, the glycol chain length further restricted the growth and orientation of Se-NPs and provided an effective alternative for the coating of the nanoparticles. The variation in the $\%$ concentrations of glycols also produced observable changes in the absorbance spectra of the prepared Se-NPs. For 

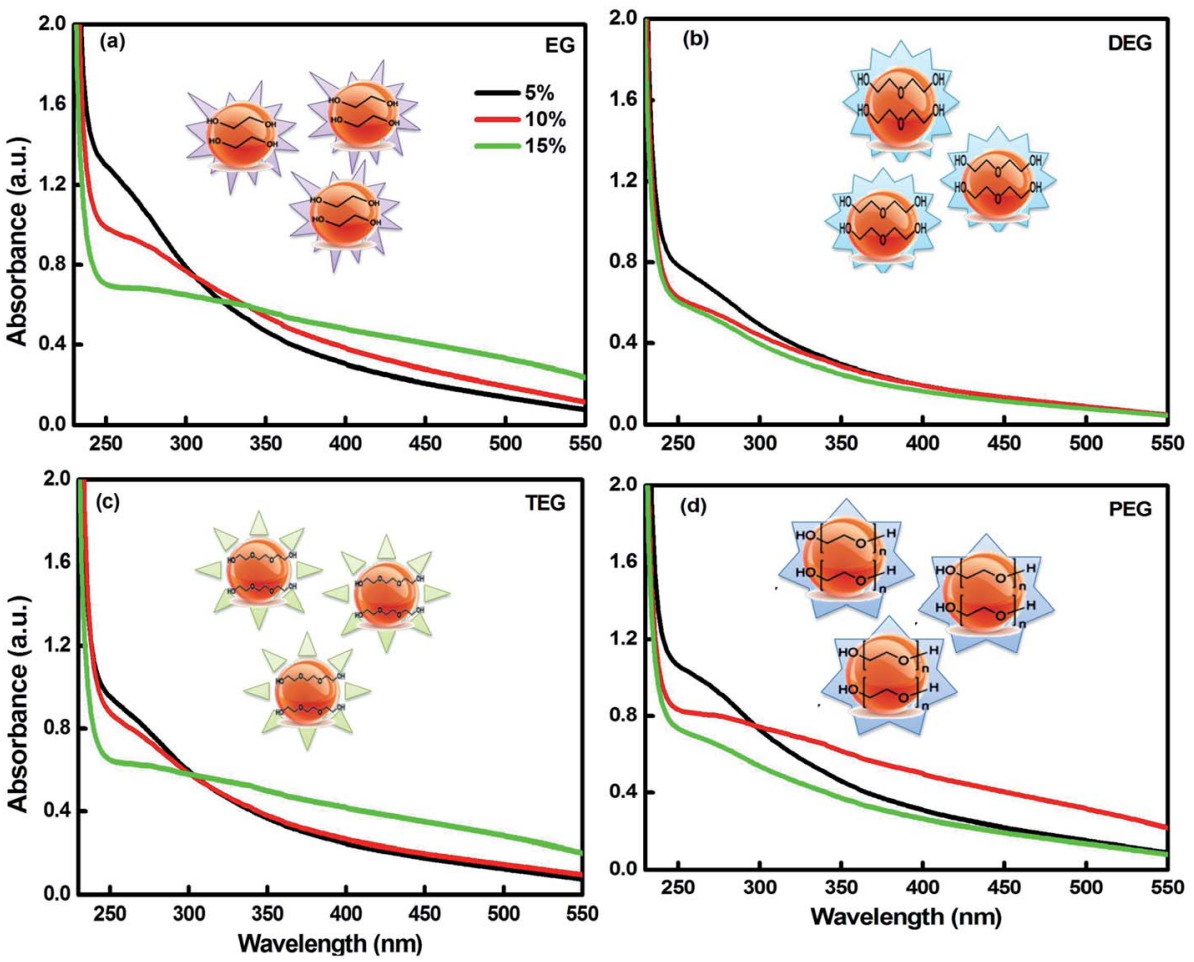

Fig. 1 UV-visible absorption spectra of (a) EG-, (b) DEG-, (c) TEG-, and (d) PEG-coated Se-NPs ([Se] = 0.03 M).

instance, on increasing the concentration of EG from 5 to $15 \%$, the absorbance values of Se-NPs displayed a hypsochromic shift in the peak. This might be due to the enhanced electrostatic interaction between EG and Se-NPs. The DEG-coated Se-NPs also displayed a similar pattern to that of the EG-coated SeNPs. On the other hand, the TEG-coated Se-NPs displayed a bathochromic shift. In case of the PEG-coated Se-NPs, a slight increment in wavelength was observed on decreasing the concentration of PEG from 15 to 5\%, whereas the pattern of optical density was similar to the remaining three cases. These variations were explained on the basis of the interaction between the glycol moieties and the formed Se-NPs.

The photo-degradation studies were accomplished for scrutinizing the effect of light on the glycol-coated Se-NPs (Fig. 2a). The time evolution spectra were also measured in the presence of light. First, UV-vis spectroscopic analysis of the freshly prepared Se-NPs was performed; their absorbance values were recorded and utilized as a control standard. Then, the samples incubated for 48 hours were removed from the chamber and their UV-vis analysis was carried out. The same experiments were repeated after 96 hours (4 days) of exposure. From the acquired absorbance values, the degradation percentage of the nanoparticles was further quantified (Fig. 2b). From the data, it was observed that after the continuous exposure of light for 72 hours, the nanoparticles were degraded by around $70 \%$ in the case of the 5\% EG-coated Se-NPs. The degradation rate started to decrease on increasing the concentration of glycols from $5 \%$ to $15 \%$. The decrease in the rate of degradation with an increase in the concentration of glycol might be due to light absorption via the presence of the strong colouration of the formed Se-NPs. From this, it was also inferred that the lower-concentration glycol-coated Se-NPs acted as a catalytic agent, whereas the high-concentration glycol-coated Se-NPs acted as a protective agent. From the environmental point of view, the degradation and decomposition of nanoparticles are very essential. If the degradation process does not take place, then, these small

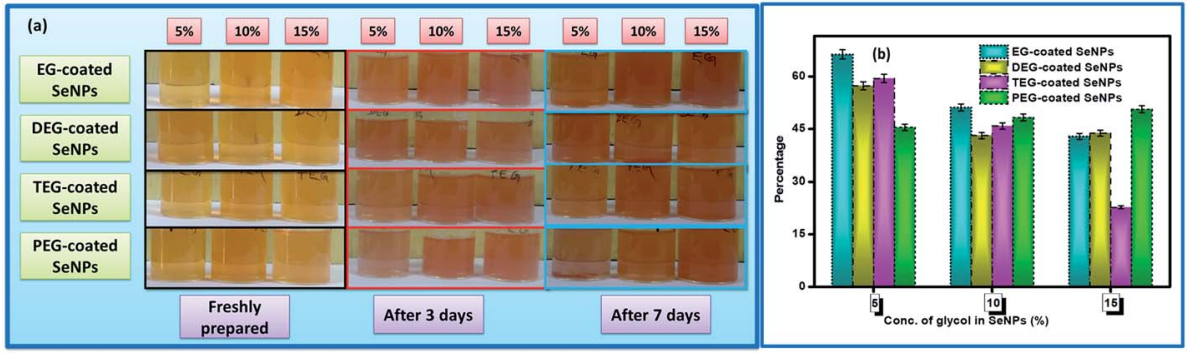

Fig. 2 (a) Digital images and (b) the corresponding percentage degradation values for the glycol-coated Se-NPs with fixed [Se] $=0.03$ M. 
particles will influence the surrounding environment, which will further lead to the disruption of different ecological processes and disturb the life cycle process. However, in our studies, the photo-degradation rate studies suggested minimum influence on the surrounding environment. Specifically, after 168 hours of exposure, it was observed that the degradation tendency of the same nanoparticles varied slightly. The negligible but significant changes indicated that the formed nanoparticles were quite stable and displayed no effect due to photo-degradation. These Se-NPs exhibited the ability to generate reactive oxygen species (ROS) during the association with an aqueous medium. In the presence of glycols, the transfer of electrons between the synthesized nanoparticles was further enhanced. The produced electrons had propensity to reduce the oxygen-generated free radical anion species such as superoxide and peroxide into hydroxyl radicals. The produced radical species further oxidised and degraded Se-NPs. The basic degradation process of Se-NPs depends on the production of electrons. The highest rate of photo-degradation for the $5 \%$ glycol-coated Se-NPs was due to the formation of additional hydroxyl radical species. On increasing the concentrations of the capping agent (5\% to $15 \%$ ), radical production was found to decrease in the system, which could be due to the strong complexation between Se and glycols. ${ }^{33,35}$ The photodegradation tendency did not decrease after 2 days, which might be due to the termination in the passivity of the electron process. The photodegradation efficiency of the glycol-coated Se-NPs could be augmented using a high-power (better $\mathrm{W}$ ) light to stimulate the surrounding.

The fluorescence emission profile displayed a characteristic band in the region from 520 to $540 \mathrm{~nm}$ (Fig. S1†). The presence of sharp peaks in the emission spectrum was mainly due to the existence of an electronic transition in Se-NPs with high photostability. The strong interaction between the glycol molecules on the surface of Se further affected the electromagnetic field around the nanoparticles and influenced the fluorescence properties of the prepared nanoparticles. FTIR analysis was performed to check the mode of attachment of the glycol molecules on the surface of Se-NPs (Fig. S2 $\dagger$ ). The broad bands at around 3392, 3346, 3325 and $3260 \mathrm{~cm}^{-1}$ corresponded to the strong - $\mathrm{OH}$ stretching due to the presence of the alcoholic group. The peaks at 2875, 2850, 2865 and $2930 \mathrm{~cm}^{-1}$ were attributed to the $\mathrm{C}-\mathrm{H}$ stretching vibration and the peaks at $1652,1635,1670$ and $1630 \mathrm{~cm}^{-1}$ were in good agreement with the $\mathrm{C}-\mathrm{C}$ stretching vibrations of the glycol molecules on the surface of Se-NPs. The bands at 1040, 1080, 1061 and $1070 \mathrm{~cm}^{-1}$ corresponded to the strong $\mathrm{C}-\mathrm{O}$ stretching in the glycol molecules. The peak at around $950 \mathrm{~cm}^{-1}$ was ascribed to the $-\mathrm{OH}$ bending. The peaks at around 890,825 and $840 \mathrm{~cm}^{-1}$ corresponded to the $=\mathrm{C}-\mathrm{H}$ bending vibrations in the glycol-coated Se-NPs. A slight variation in the intensities and wavelength positions was observed in the FTIR spectra of the $5 \%, 10 \%$ and $15 \%$ glycol-coated Se-NPs, which may be due to the change in the concentration of glycols on Se-NPs. In addition, the absence of any other type of functional group peak further suggested the purity and perfect orientation of the glycol-coated Se-NPs. The results further suggested the better adsorption of glycols on the surface of the nanoparticles. This study clearly demonstrated that glycols have a tendency to interact with the Se ions during the synthesis of nanoparticles and simultaneously stabilize the size and shape of the particles.

The morphological characteristics of the glycol-coated SeNPs were visualized using HRTEM (Fig. 3). The particles were found to be dispersed and nearly spherical in shape with sizes ranging between 15 and $40 \mathrm{~nm}$. These glycol molecules had the potential to adsorb on the surface of Se-NPs and modulate the size and orientation of the formed Se-NPs. The slight differences in the sizes of Se-NPs for different types of the chosen glycol molecules were associated with the different interacting abilities of the glycols with the formed nanoparticles.

It was further observed that the agglomeration tendency was minimum for the EG- and DEG-coated Se-NPs. The small chain lengths of EG and DEG resulted in better surface coverage and higher stabilizing ability towards Se-NPs. The enhancement in viscosity with the chain length for TEG and PEG resulted in lesser surface coverage on Se-NPs and larger-sized nanoparticles. The mobilizing ability of TEG and PEG was not appropriate for the stabilization of the formed Se-NPs, resulting in higher agglomeration tendency of particles. The size of the formed nanoparticles was further investigated via DLS studies as a function of different concentrations of the chosen glycols (Table S1 $\dagger$ ). From the data, it was observed that the size of SeNPs displayed an inverse relationship with the concentration of all the chosen glycols. There was an observable decrease in the size of the nanoparticles on enhancing the amount of glycols from $5 \%$ to $15 \%$, which was related to the prevention of the agglomeration of Se-NPs by the glycols. The higher concentration of glycols avoided the direct association of the nanoparticles and controlled their aggregation and provided SeNPs with uniform morphology and size. On comparing the types of the chosen glycols, it was found that the maximum size and highest PDI value were obtained in the case of the TEGcoated Se-NPs due to their highest rate of agglomeration, whereas the EG-coated Se-NPs produced smaller-sized nanoparticles.

The thermal behaviour of the glycol-capped Se-NPs was investigated via TG-DTA analysis in the temperature range of 0$1000^{\circ} \mathrm{C}$ under a nitrogen gas atmosphere (Fig. S3 $\dagger$ ). Two major weight losses were observed during the heating process in the case of the EG-coated Se-NPs. The first weight loss of 7\% was observed between 230 and $250^{\circ} \mathrm{C}$, which was mainly associated with the evaporation of moisture. In addition, continuous and relative $71 \%$ weight loss was further observed on increasing the temperature beyond $250{ }^{\circ} \mathrm{C}$. This was mainly associated with the decomposition, oxidation and reduction reactions occurring on the surface of the synthesized nanomaterials. In case of the DEG-coated Se-NPs, three major weight losses were observed. The first weight loss occurred at $100{ }^{\circ} \mathrm{C}$ with $8 \%$ loss, which was mainly associated with the evaporation of the moisture and water content present in the synthesized nanoparticles. The decomposition of the glycol moieties occurred between 200 and $500{ }^{\circ} \mathrm{C}$. The first weight loss of around $25 \%$ occurred at $200{ }^{\circ} \mathrm{C}$ and the second $65 \%$ loss occurred between 200 and $500{ }^{\circ} \mathrm{C}$. Similarly, in the case of the TEG- and PEG- 


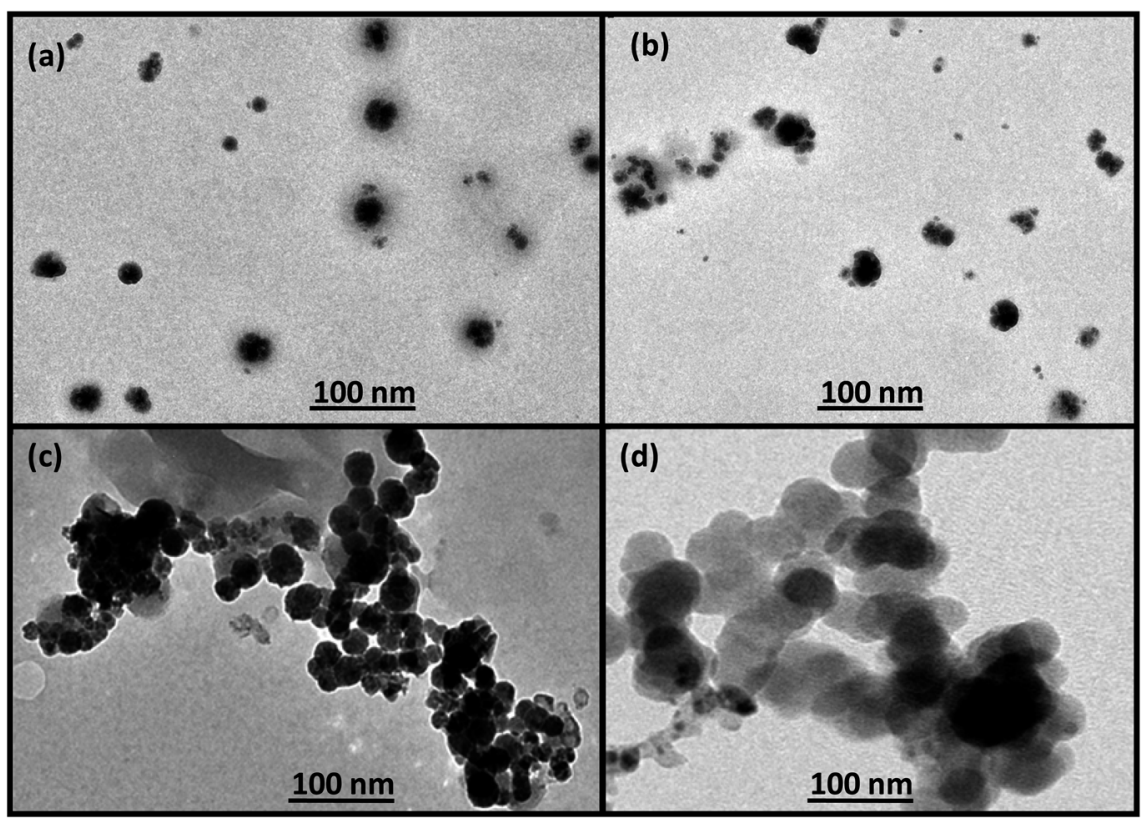

Fig. 3 HRTEM images of (a) EG-, (b) DEG-, (c) TEG- and (d) PEG-coated Se-NPs (the concentration of Se was fixed at 0.03 M).

coated Se-NPs, three weight losses were observed. The first weight loss of $12 \%$ occurred at $100{ }^{\circ} \mathrm{C}$, followed by $46 \%$ weight loss at $250{ }^{\circ} \mathrm{C}$. In the final step, $40 \%$ weight loss occurred at $500{ }^{\circ} \mathrm{C}$. In the case of the PEG-coated Se-NPs, first, $9 \%$ weight loss occurred at $100{ }^{\circ} \mathrm{C}$, followed by $57 \%$ weight loss at $400{ }^{\circ} \mathrm{C}$; in the final step, $32 \%$ weight loss occurred at $450{ }^{\circ} \mathrm{C}$.

In the DTA thermogram, one endothermic peak was observed in the case of the EG-capped Se-NPs due to one major weight loss, whereas in the case of the DEG-, TEG- and PEGcoated Se-NPs, three endothermic peaks were observed. From the above data, it could be inferred that the EG-coated Se-NPs were the least thermally stable than the DEG-, TEG- and PEGcoated Se-NPs. The behaviour of TGA was found to be reproducible, which gave complete information about the decomposition of the glycol-coated Se-NPs and suggested the thermal stability of the as-formed Se-NPs. DSC analysis was performed to determine the amount of heat released or absorbed by the glycol-coated Se nanoparticles. The presence of a single peak for each type of Se-NPs reflected the existence of a single crystalline phase of the particles during the heating process. The obtained curves represented in Fig. S4† further suggest the exothermic nature and purity of the glycol-coated Se-NPs. In case of the EG-, DEG-, TEG- and PEG-coated Se-NPs, the values of enthalpy were found to be $42.92,209.2,172.9$ and $54.93 \mathrm{~J} \mathrm{~g}^{-1}$, whereas the values of transition temperature were found to be 221.18, 220.06, 223.22 and $221.86{ }^{\circ} \mathrm{C}$, respectively. The values of onset temperature, glass transition temperature and melting temperature were found to be $209.19,219.19$ and $222.41{ }^{\circ} \mathrm{C}$ for the EG-coated Se-NPs, $190.06,220.06$ and $221.90^{\circ} \mathrm{C}$ for the DEGcoated Se-NPs, $211.93,223.22$ and $224.56^{\circ} \mathrm{C}$ for the TEG-coated Se-NPs and $217.58,221.86$ and $225.89^{\circ} \mathrm{C}$ for the PEG-coated SeNPs, respectively. From the obtained data, it was very clear that the synthesized nanoparticles have an exothermic nature with high thermal stability. To explore the purity of the synthesized EG-, DEG-, TEG- and PEG-coated Se-NPs, energy dispersive X-ray (EDX) spectroscopic analysis was also carried out (Fig. S5 $\dagger$ ). The developed Se-NPs exhibited an absorption band between 0 and $2 \mathrm{keV}$ for $\mathrm{C}, \mathrm{O}, \mathrm{Na}$ and $\mathrm{Se}$, whereas an additional band was observed at $11 \mathrm{keV}$ for Se. The EDX spectrum reflects the peaks of the major constituents such as $\mathrm{Se}, \mathrm{O}, \mathrm{Na}$ and $\mathrm{C}$, and no additional peaks was observed, which further confirms the formation of pure glycol-coated Se-NPs. The peaks for the C and $\mathrm{O}$ elements in the spectrum confirmed the presence of glycol molecules on the surface of Se-NPs.

Powder X-ray diffraction studies were carried out to scrutinize the crystalline nature and effect of the glycol coating on the surface of Se-NPs (Fig. S6 $\dagger$ ). The major peaks were observed at $2 \theta=23.53,29.79,41.05,43.60,45.43,51.70,55.94,61.46$ and $65.21^{\circ}$ for the EG-, DEG-, TEG- and PEG-coated Se-NPs, which corresponded to the (100), (101), (110), (102), (111), (201), (202), (031) and (210) diffraction planes in the surface-functionalized Se-NPs. The crystallite sizes calculated using the Debye-Scherrer equation were found to be 7, 7.3, 8.8 and $9.6 \mathrm{~nm}$ for the EG-, DEG-, TEG- and PEG-coated Se-NPs, respectively. All the chosen types of glycols produced similar diffraction planes for Se-NPs. However, one extra peak was observed in the case of the TEGand PEG-coated Se-NPs at $2 \theta=68.34^{\circ}$, which corresponded to the (211) diffraction plane. The crystalline peak for bare Se-NPs was found to be comparable with that of the glycol-coated SeNPs. ${ }^{36}$ Accordingly, it could be inferred that glycols fully adhered on the surface of Se-NPs and did not affect the crystalline plane of the formed nanoparticles. The slight broadening in the diffraction peaks was associated with morphological alteration, which resulted due to the existence of a mixture of orthorhombic and tetragonal geometries. In the case of the TEG- and PEG-coated Se-NPs, the variations in the 
diffraction peaks of Se-NPs originated from the overshadowing of the peaks in the presence of TEG and PEG. The presence of a large number of oxygen molecules in the TEG and PEG chains resulted in the coordination saturation of the dangling bonds on the surface of Se-NPs and hence their lower stabilization. The presence of TEG and PEG further reduced the active sites for reduction on the surface of Se-NPs. Raman spectroscopy has been considered as the most sensitive technique to differentiate between the assorted allotropic and crystalline forms of selenium present in Se-NPs (Fig. S7 $\dagger$ ). The presence of a strong band at $234 \mathrm{~cm}^{-1}$ confirmed the formation of trigonal selenium (t-Se) from the phase transformation of amorphous selenium along with the chain-like structure of t-Se. The introduction of glycols as a surface decorating agent during synthesis resulted in the generation of one new broad band near $450 \mathrm{~cm}^{-1}$. The formation of the new peak might be due to the presence of the hydroxyl groups in the ethylene, diethylene, triethylene and polyethylene glycols. Similar results were obtained for all four types of glycol-coated Se-NPs.

\section{Antimicrobial activities of glycol-coated Se-NPs}

The in vitro antimicrobial activities of glycol-coated Se-NPs were investigated using Gram negative (E. coli, P. aeruginosa and $S$. typhi) and Gram positive ( $S$. aureus) bacteria. This study demonstrated the temperamental effect of the as-synthesized Se-NPs on the inhibitory growth of bacteria. Three different concentrations of glycols, i.e., 5\%, 10\% and $15 \%$ were used to study the respective activities. The absence of a zone of inhibition for the E. coli, S. aureus, and $S$. typhi bacteria in the presence of all four types of the glycol-coated Se-NPs suggested the absence of any kind of antibacterial activity in these nanoparticles (Fig. 4).

Here, in this study, deionised water was used as a standard control system. The growth of bacteria in the presence of the glycol-modified Se-NPs was compared with that for the control to calculate the value of the zone of inhibition. The prepared SeNPs were found to be biocompatible and non-toxic towards these bacterial strains. The presence of glycol-coated Se-NPs produced no effect on the hydrogen-containing species such as methanogens and sulphates in the case of $E$. coli and they were found to be biocompatible towards this bacterium strain. In the case of $S$. aureus, the cell wall is made up of peptidoglycans (group of differently bridged peptides); it is infective and pathogenic in nature and can easily get colonized. $S$. aureus can also act symbiotically in nature in the presence of Se-NPs. Due to this reason, the DNA of the cell wall of $S$. aureus was not

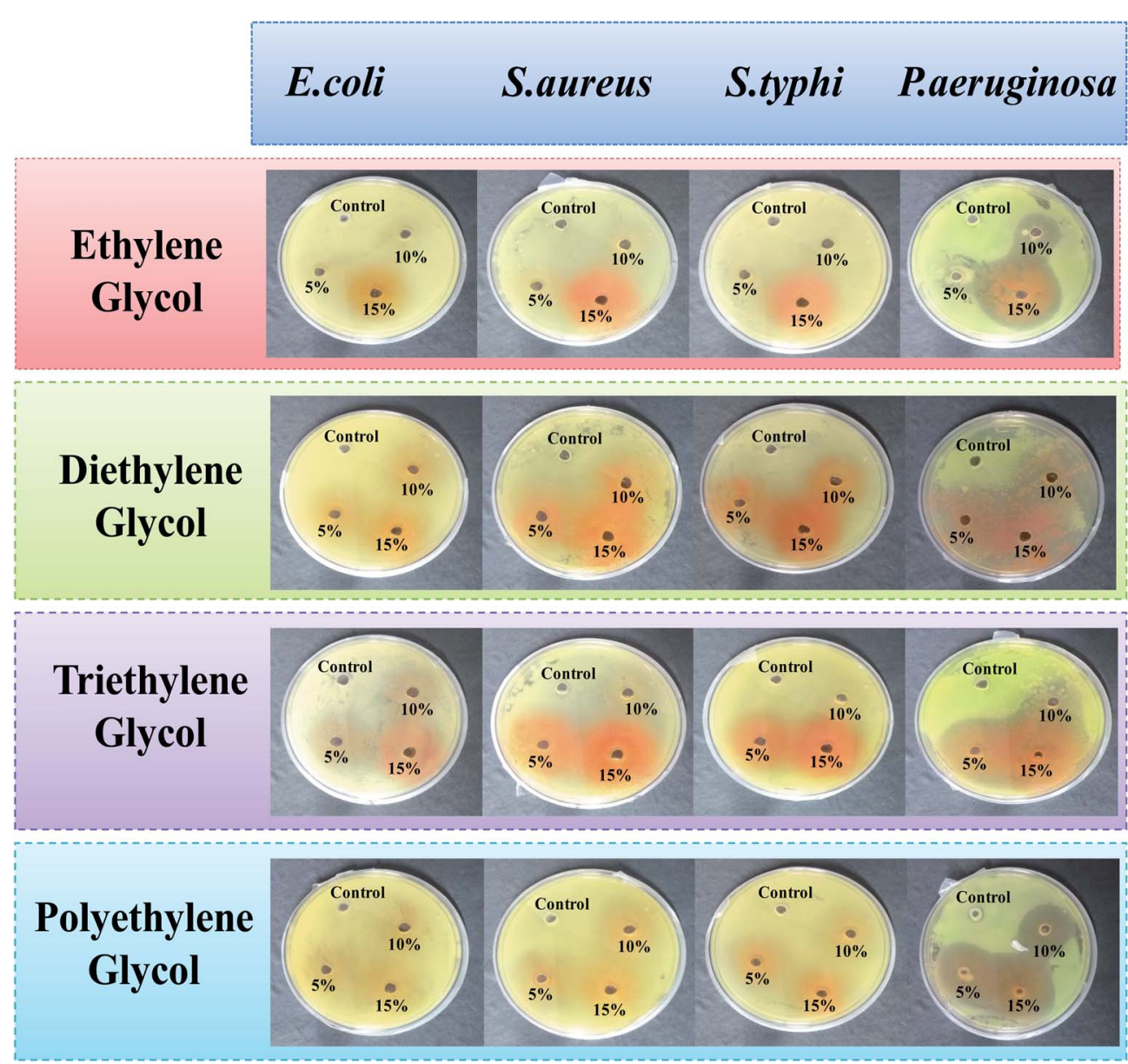

Fig. 4 Antimicrobial activity of the EG-, DEG-, TEG- and PEG-coated Se-NPs (0.03 M Se) as a function of different concentrations of glycol on the $E$. coli, $P$. aeruginosa, S. typhi and $S$. aureus bacterial strains. 
ruptured in the presence of the glycol-coated Se-NPs and the particles were biocompatible with this bacterial strain. A similar behaviour was observed for $S$. typhi in the presence of Se-NPs. In the case of $P$. aeruginosa, all the glycol-coated Se-NPs exhibited antibacterial activity. The region of the zone of inhibition was found to be enhanced with the respective increase in the concentration of glycols. Thus, it was also concluded that all the chosen concentrations of EG-, DEG-, TEG- and PEG-coated SeNPs exhibited high and strong selectivity towards $P$. aeruginosa. The selectivity of the different glycol-coated Se-NPs towards $P$. aeruginosa might be explained on the basis of the cell wall structure. The presence of phosphorous, fatty acids, nitrogen, lipids, lipopolysaccharides and proteins in the cell resulted in high affinity towards Se-NPs. In addition, the outer membrane of $P$. aeruginosa is very thin; which resulted in the permeability of Se-NPs in the cell wall, affected the internal functioning of the bacterial activities and retarded its growth. The enhanced concentrations of glycol on the surface of Se-NPs produced smaller-sized Se-NPs. As a result, more number of particles entered the bacterial cell wall and produced a biggersized zone of inhibition. From the above discussion, it was concluded that the EG-, DEG-, TEG- and PEG-coated Se-NPs were biocompatible towards E. coli, S. aureus and S. typhi and simultaneously highly selective and sensitive towards $P$. aeruginosa.

The maximum concentration of each glycol, i.e., 15\% was chosen for the in vitro stability analysis of the glycol-coated SeNPs. Here, $15 \%$ glycol-coated Se-NPs were used as the standard control system. The values of optical density of the glycol-coated Se-NPs were compared with the optical density values of different blood components present in the glycol-coated Se-NPs. The results were obtained by determining the effect of the glycol-coated Se-NPs in contact with $2 \%$ BSA, $0.2 \mathrm{M}$ histidine and cysteine. Also, a $0.9 \% \mathrm{NaCl}$ solution and phosphate buffer $(\mathrm{pH}=5.6,6.4,7$ and 7.5) were used for the measurement (Fig. 5).

On interpreting the data, it was found that the absorption hump at $238 \mathrm{~nm}$ showed an observable hypochromic peak shift of $8 \mathrm{~nm}$ in the presence of different types of blood components, which demonstrated that the glycol-coated SeNPs were stable and intact. In addition, the aggregation of the particles was observed in the presence of cysteine. The presence of buffer solutions with different $\mathrm{pH}$ values $(5.6,6.4$, 7 and 7.5) also produced a hypochromic shift in the wavelength of the glycol-coated Se-NPs (Fig. S8†), suggesting the stability of glycol-coated Se-NPs in all the tested blood components. In the presence of DMEM and PBS, the glycolcoated Se-NPs exhibited a similar behaviour to pure $15 \%$ glycol-coated Se-NPs with a slight hypsochromic shift. The slight blue shift in the absorption spectrum suggested the formation of more stable Se-NPs. In the case of HSA, a new peak was observed at $280 \mathrm{~nm}$ for the glycol-coated Se-NPs. This was due to the presence of HSA in the developed Se-NPs (Fig. S9 $\dagger$ ). The average hydrodynamic diameter of the $15 \%$ glycol-coated Se-NPs was calculated through DLS measurements and presented in Table S2. $\dagger$

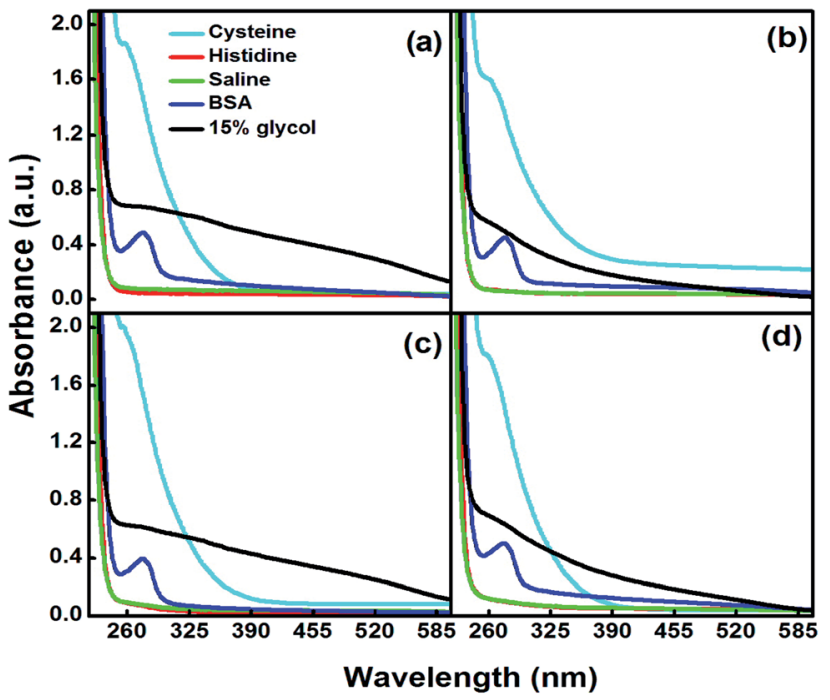

Fig. 5 In vitro stability of $15 \%$ (a) EG-, (b) DEG-, (c) TEG- and (d) PEGcoated Se-NPs $(0.03 \mathrm{M})$ in the presence of various blood components.

\section{Antioxidant activity of glycol-coated Se-NPs}

Antioxidant properties have the potential for the diverse implementation of the chosen materials in science and provide information about the inhibition of free radical species. Se particles are considered as one of the active elements in living systems and therefore, their anti-oxidative activities need a thorough investigation. In the current study, ascorbic acid was chosen as the control standard due to its maximum antioxidant activities, and its value of absorbance was utilized to calculate the antioxidant activity of the glycol-capped Se-NPs (Fig. 6a). ${ }^{26,27}$ All the chosen Se-NPs displayed a variation in their orange coloration in the presence of the antioxidant reagent. The visual confirmation of the antioxidant activity of the studied Se-NPs was done by the transformation of the orange color to a bluish-green color. On interpreting the results, it was found that the $15 \%$ EG-coated Se-NPs were more potent in showing antioxidant activity. The antioxidant activity of the glycol-coated Se-NPs displayed enhancement with an increase in the concentration of the glycol coating over the surface of Se-NPs. This was mainly due to the decrease in the size of the formed nanoparticles via the higher rate of the encapsulation ability of glycols at a higher concentration. The smaller-sized particles possess a high surface area for more interactions with antioxidant agents. In addition, the glycol group possesses a sufficient amount of hydroxyl groups, due to which they can reduce the activity of free radical induced oxidative species. The scavenging activity and ability to inhibit oxidation were checked for the developed nanoparticles utilizing hydrogen peroxide free radical activity. The absorbance values of the respective nanoparticles were recorded at $235 \mathrm{~nm}$ (Fig. 6c) and furthermore, the reducing ability of Se-NPs (Fig. 6b) was examined. ${ }^{30,31}$ Out of the four chosen glycol coatings, the EG- and PEG-coated Se-NPs showed the maximum total reducing scavenging activity (Fig. 6b). The presence of Se and more-OH groups from PEG on the surface of the nanoparticles resulted in the tendency to 

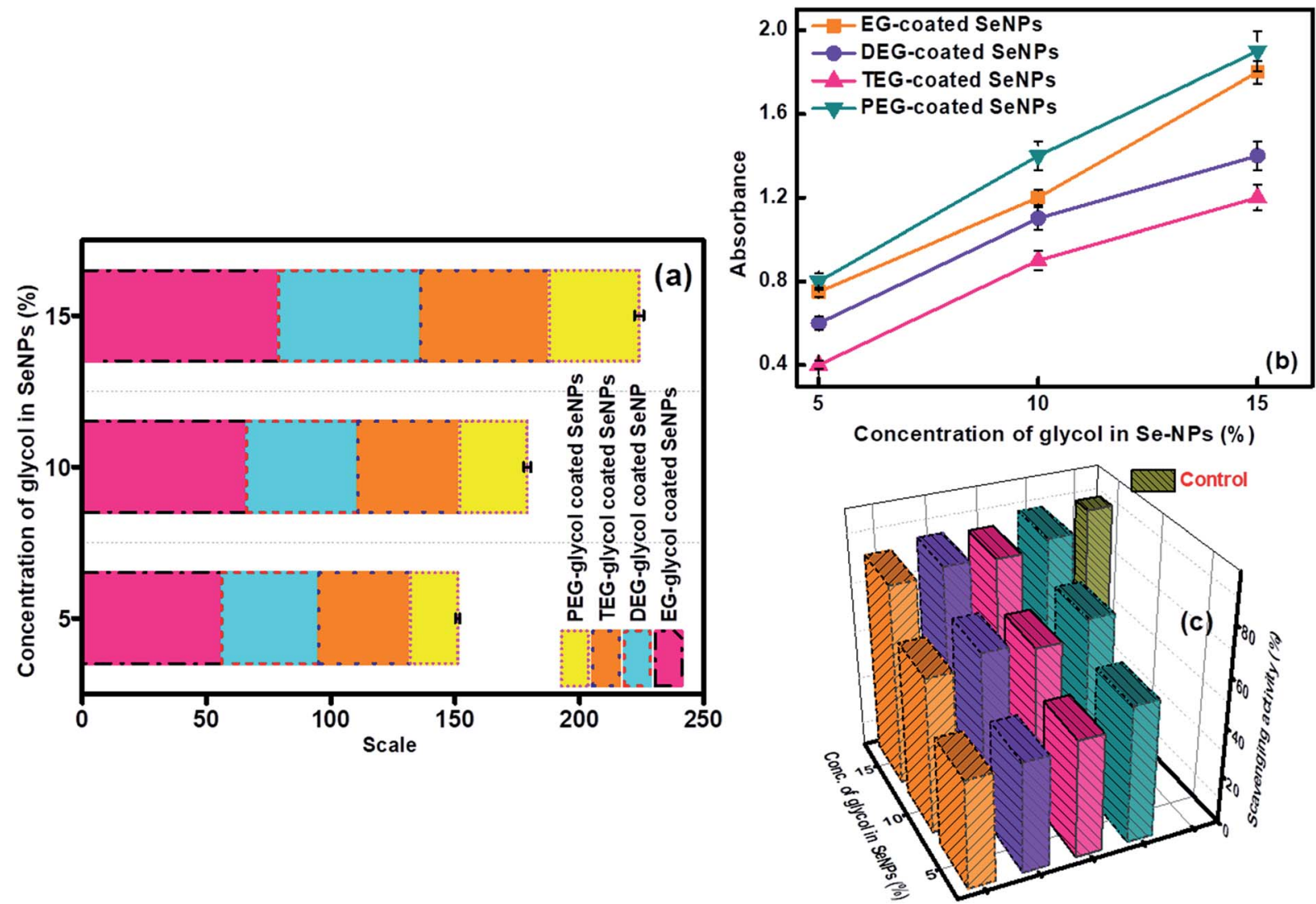

Fig. 6 (a) Antioxidant property, (b) total reducing ability and (c) hydrogen peroxide free radical scavenging activity for glycol-coated Se-NPs with $[\mathrm{Se}]=0.03 \mathrm{M}$.

break or rupture the weak peroxide bond. The maximum hydrogen peroxide scavenging activity was observed for the $15 \%$ glycol-coated Se-NPs (Fig. 6c). On comparing the data of all the above-mentioned biological activities, it was inferred that the glycol-coated Se-NPs were biocompatible in the permissible limits. The 5, 10 and 15\% glycol-coated Se-NPs were found to be highly biocompatible in antimicrobial activity and highly selective towards $P$. aeruginosa bacteria. With respect to antioxidant activity, hydrogen peroxide free radical scavenging activity and total reducing activity, it was observed that on increasing the concentration of glycols from 5 to $15 \%$, the value of optical density increased, which led to an increment in the antioxidant behaviour of Se-NPs; the PEG-coated Se-NPs were found to be the most effective. Similarly, in the in vitro study, 15\% PEG-coated Se-NPs were found to be highly intact in all the biological components. Thus, it can be concluded that the respective nanoparticles exhibit consistent results in all the biological studies.

\section{Aggregation and dissolution behaviour of glycol-coated Se- NPs}

The intensity weighing pattern of the hydrodynamic radii was chosen by using the DLS technique to determine the size distribution profiles of Se-NPs using the diffusion-limited colloidal aggregation theory (DLCA). Here, the effect of $\mathrm{pH}$ and ionic strength on the aggregation and dissolution behaviour of the $15 \%$ glycol-coated Se-NPs was chosen for the studies. From the DLS analysis, it was found that $\mathrm{pH}$ played a significant role in the protonation, deprotonation and electron-donating ability of the nanoparticles. On increasing the $\mathrm{pH}$ of the solution from 2 to 10, the size of the glycol-coated Se-NPs decreased and the minimum size was obtained at $\mathrm{pH}=$ 10 (Fig. 7b). The PDI value was found to be 0.3 in the case of $\mathrm{pH}$ $=10$, which further suggested the uniform and monodispersed nature of the synthesized nanoparticles. However, after increasing the $\mathrm{pH}$ of the nanoparticle solution from 10 to 12, the size and PDI started to increase. The results reflected that the maximum sizes of 408, 375, 396 and $382 \mathrm{~nm}$ for the 15\% EG-, DEG-, TEG- and PEG-coated Se-NPs were observed at $\mathrm{pH}=2$. The minimum sizes of 46, 58, 94 and $76 \mathrm{~nm}$ for the 15\% EG-, DEG-, TEG- and PEG-coated Se-NPs were observed at $\mathrm{pH}=10$ (Fig. 7b). Thus, it can be concluded that the maximum and minimum agglomeration tendencies were observed at $\mathrm{pH}$ $=2$ and $\mathrm{pH}=10$, respectively. At $\mathrm{pH}=2$, the presence of a high concentration of $\mathrm{H}^{+}$ions made the surface more passive in nature. The high concentration of $\mathrm{H}^{+}$ions combined with the water molecules present in the aqueous media of nanoparticles, resulting in the formation of hydronium ions. These hydronium ions further leads to the formation of various hydrospheres around the exterior of Se-NPs, which caused the 


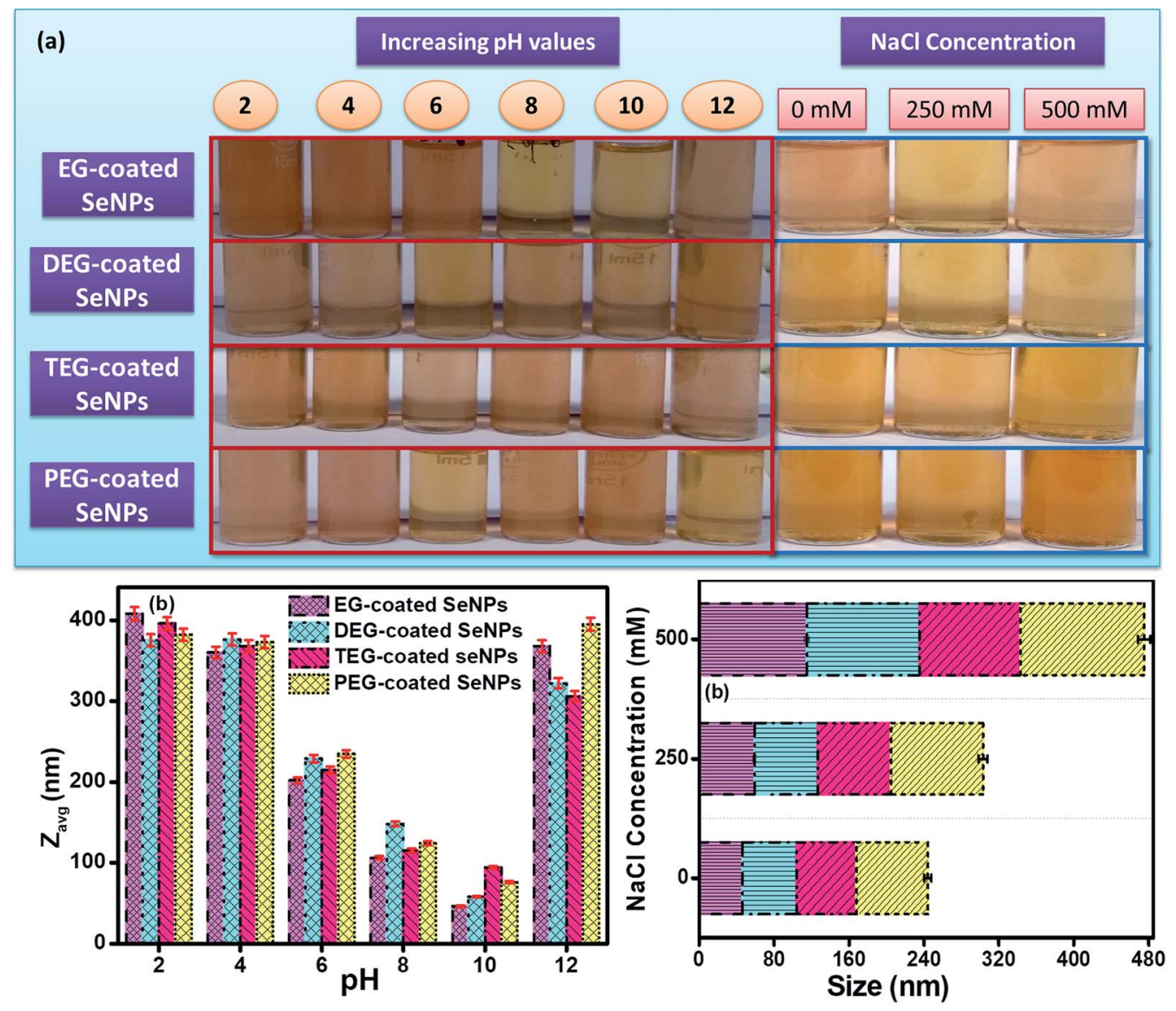

Fig. 7 (a) Digital images showing the effect of aggregation and dissolution behaviour, with the respective effect on size as a function of (b) pH and (c) concentration of $\mathrm{NaCl}$ in the presence of glycol-coated Se-NPs (concentration of Se kept fixed at $0.03 \mathrm{M}$ ).

aggregation of the nanoparticles and formed large-sized particles. Simultaneously, at acidic $\mathrm{pH}$, the $-\mathrm{OH}$ group present on each glycol restricted the electron-donating ability. At $\mathrm{pH}=12$, the sizes of the encapsulated Se-NPs were again found to increase. This was due to the formation of hydronium ions at a high basic $\mathrm{pH}$ (due to the availability of a large amount of $\mathrm{OH}^{-}$ions), which further produced hydrospheretype aggregations in the nanoparticles. In addition, the alcohol group has the tendency to convert into hydroxyl ions due to its high electron-donating ability in an alkaline medium. At $\mathrm{pH}=10$, the synthesized nanoparticles were surrounded by a few hydroxyl ions, which caused strong repulsion around the system and prevented the agglomeration of the particles. Thus, it was also inferred that the glycolcoated Se-NPs were more stable and played a pivotal role at $\mathrm{pH}=10$. The aggregation tendency of the glycol-coated Se-NPs was also investigated in the presence of the solutions of different ionic strengths $(0,250$ and $500 \mathrm{mM})$. On increasing the ionic strength from 0 to $500 \mathrm{mM}$, the size of the nanoparticles was found to increase to a greater extent (Fig. 7c). The high absorption of ions on the surface of the nanoparticles was due to the existence of more number of ions $\left(\mathrm{Na}^{+}\right.$and $\left.\mathrm{Cl}^{-}\right)$ at $500 \mathrm{mM}$. The electrostatic interaction further caused the adsorption of these ions, which led to the aggregation of the synthesized Se-NPs. In addition, the higher ionic strength of the solution resulted in the suppression of the electrostatictype of interactions and produced agglomeration in Se-NPs. Additional data on the comparison of bare (naked), surfactant-capped and glycol-capped Se-NPs were also obtained to investigate the influential behaviour of these nanoparticles on basic environmental species (Table S3†). The special effect of the glycol-coated Se-NPs was observed compared to that for other nanoparticles, which suggested that the glycol-coated Se-NPs exhibited a more biocompatible nature as compared to the surfactant-capped Se-NPs, whereas naked Se-NPs were found to be the most toxic. ${ }^{37}$

\section{Sorption behaviour of glycol-coated Se-NPs}

Sorption studies were performed to investigate the influential behaviour of the glycol-coated Se-NPs on soil and aquatic species (Fig. S10†). For this analysis, the high sorption ability of loamy soil was used. The sorption capacity further depends on the organic carbon and clay content present in the soil. For measurements, freshly prepared glycol-coated Se-NPs were used as the control or reference set-up and their absorbance values were recorded via UV-vis spectroscopy. Subsequently, the nanoparticles mixed with soil samples were utilized after their 
incubation period for analysis. Their absorbance values were noted for data interpretation. These absorbance values were further utilized for calculating the adsorption tendency of the glycol-coated Se-NPs in soil and compared with that of freshly prepared glycol Se-NPs. From the obtained data, it was observed that the absorbance value decreased with a slight bathochromic shift in wavelength. This variation was due to the reduction in the agglomeration tendency of the particles. The decreased absorption value was due to the immobilization of the glycolcoated Se-NPs in the soil. The percentage of nanoparticles left in the supernatant was calculated and shown in Fig. 8 for each type of glycol-coated Se-NPs. From the data, it was found that around $70 \%$ of the nanoparticles were sorbed by the soil in the case of the $5 \%$ glycol-coated Se-NPs. Only $30 \%$ of the nanoparticles were left in the supernatant. The greater sorption capacity, mineral composition and expeditious uptake further led to more number of sorption sites in the soil due to the destruction of aggregation. The above results demonstrated that the maximum sorption capacity by soil was found for the $5 \%$ glycol-encapsulated Se-NPs. This further reduced the toxicity with the consumption of these nanoparticles and enhanced their environmental fate. Therefore, it was proposed that glycol coated-Se-NPs in contact with the environment and hydrosphere will be easily absorbed by the abyssopelagic layer and are thus considered biocompatible to the living species. The minimum sorption was observed in the case of the $15 \%$ glycol-coated Se-NPs due to the formation of strong and stable Se-NPs. This was mainly due to the full adherence of $15 \%$ glycols on the Se surface. The slow diffusion process of sorbate in the loamy soil further led to slow degradation. The transformation of the glycol-coated Se-NPs was also studied in aqueous media, and the results are shown in Fig. S11. $\dagger$ From the data, it was concluded that the $15 \%$ glycol-coated Se-NPs were the most effective with a minimum concentration remaining in the aqueous solution. Out of the four types of glycol-coated Se-NPs, the PEG-coated Se-NPs were found to be less effective in an aqueous medium.

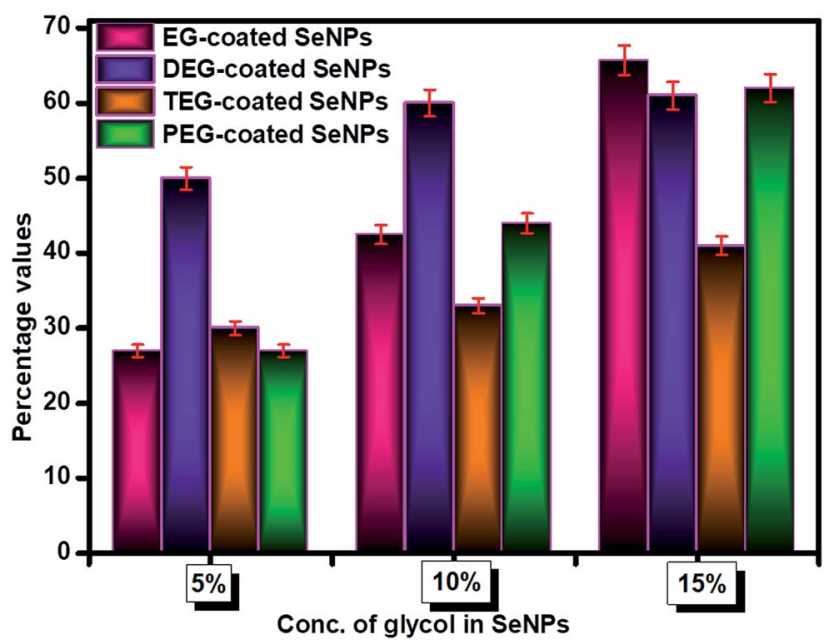

Fig. 8 Percentage of glycol-coated Se-NPs (0.03 M) in supernatant after soil-sorption experiment.

\section{Conclusion}

Herein, the facile chemical synthesis of Se-NPs was carried out by using EG, DEG, TEG and PEG as encapsulating agents. The synthesized glycol-capped Se-NPs exhibited strong absorption and emission properties. The antimicrobial activity results for the developed nanoparticles demonstrated that they were highly susceptible and discriminatory towards the $P$. aeruginosa bacteria. The in vitro study clearly demonstrated that the synthesized nanoparticles were highly compatible in nature and did not affect any component of blood except L-cysteine. The antioxidant performance, hydrogen peroxide free radical scavenging activity and total reducing capability of the synthesized nanoparticles were evaluated, which showed that the EG-coated Se-NPs have the maximum efficiency. The environmental fate assessment of the developed Se-NPs was carried out via soil sorption activity. The $5 \%$ glycol coated Se-NPs were found to be more potent in the sorption study. A photodegradation experiment was also carried out to investigate the stability of Se-NPs, which further suggested that the $5 \%$ glycol-coated Se-NPs were the most stable. The aggregation and dissolution behaviours were also checked, which confirmed that the synthesized nanoparticles were stable at $\mathrm{pH}=10$ (a slightly alkaline medium).

\section{Conflicts of interest}

There are no conflicts of interest to declare.

\section{Acknowledgements}

Savita Chaudhary is thankful to DST Inspire Faculty award [IFACH-17] and DST Purse grants II for financial assistance. Authors are highly thankful to SAIF CIL Panjab University for technical assistance.

\section{References}

1 S. Menon, K. S. S. Devi, H. Agarwal and V. K. Shanmugam, Efficacy of Biogenic Selenium Nanoparticles from an Extract of Ginger towards Evaluation on Anti-Microbial and Anti-Oxidant Activities, Colloid Interface Sci. Commun., 2019, 29, 1-8.

2 W. Y. Qiu, Y. Y. Wang, M. Wang and J. K. Yan, Construction, stability, and enhanced antioxidant activity of pectindecorated selenium nanoparticles, Colloids Surf., B, 2018, 170, 692-700.

3 S. Menon, S. Devi KS, R. Santhiya, S. Rajeshkumar and V. Kumar, Selenium nanoparticles: A potent chemotherapeutic agent and an elucidation of its mechanism, Colloids Surf., B, 2018, 170, 280-292.

4 D. Chakraborty, P. Chauhan, S. A. Alex, S. Chaudhary, K. R. Ethiraj, N. Chandrasekaran and A. Mukherjee, Comprehensive study on biocorona formation on functionalized selenium nanoparticle and its biological implications, J. Mol. Liq., 2018, 268, 335-342. 
5 H. Sardarabadi, M. Mashreghi, K. Jamialahmadi, M. M. Matin and M. Darroudi, Selenium nanoparticle as a bright promising anti-nanobacterial agent, Microb. Pathog., 2019, 126, 6-13.

6 A. Khurana, S. Tekula, M. A. Saifi, P. Venkatesh and C. Godugu, Therapeutic applications of selenium nanoparticles, Biomed. Pharmacother., 2019, 111, 802-8012.

7 L. C. Tan, Y. C. Nancharaiah, E. D. Hullebusch and P. N. L. Lens, Selenium: environmental significance, pollution, and biological treatment technologies, Biotechnol. Adv., 2016, 34, 886-907.

8 T. M. Sakr, M. Korany and K. V. Katti, Selenium nanomaterials in biomedicine-An overview of new opportunities in nanomedicine of selenium, J. Drug Delivery Sci. Technol., 2018, 46, 223-233.

9 H. Zhang, Q. Sun, L. Tong, Y. Hao and T. Yu, Synergistic combination of PEGylated selenium nanoparticles and $\mathrm{X}$ ray-induced radiotherapy for enhanced anticancer effect in human lung carcinoma, Biomed. Pharmacother., 2018, 107, 1135-1141.

10 Q. Xie, W. Deng, X. Yuan, H. Wang, Z. Ma, B. Wu and $\mathrm{X}$. Zhang, Selenium-functionalized liposomes for systemic delivery of doxorubicin with enhanced pharmacokinetics and anticancer effect, Eur. J. Pharm. Biopharm., 2018, 122, 87-95.

11 U. Luesakul, S. Komenek, S. Puthong and N. Muangsin, Shape-controlled synthesis of cubic-like selenium nanoparticles via the self-assembly method, Carbohydr. Polym., 2016, 153, 435-444.

12 H. Li, D. Liu, S. Li and C. Xue, Synthesis and cytotoxicity of selenium nanoparticles stabilized by $\alpha$-D-glucan from Castanea mollissima Blume, Int. J. Biol. Macromol., 2019, 129, 818-826.

$13 \mathrm{X}$. Ye, L. Chen, L. Lui and Y. Bai, Electrochemical synthesis of selenium nanoparticles and formation of sea urchin-like selenium nanoparticles by electrostatic assembly, Mater. Lett., 2017, 196, 381-384.

14 S. Chaudhary and S. K. Mehta, Selenium Nanomaterials: Applications in Electronics, Catalysis and Sensors, J. Nanosci. Nanotechnol., 2014, 14, 1658-1674.

15 Y. Huang, L. He, W. Liu, C. Fan, W. Zheng, Y. S. Wong and T. Chen, Selective cellular uptake and induction of apoptosis of cancer-targeted selenium nanoparticles, Biomaterials, 2013, 34, 7106-7116.

16 S. Nath, S. K. Ghosh, S. Panigahi, T. Thundat and T. Pal, Synthesis of Selenium Nanoparticle and Its Photocatalytic Application for Decolorization of Methylene Blue under UV Irradiation, Langmuir, 2004, 20, 7880-7883.

17 K. Kalishwaralal, S. Jeyabharathi, K. Sundar, S. Selvamani, M. Prasanna and A. Muthukumaran, A novel biocompatible chitosan-Selenium nanoparticles (SeNPs) film with electrical conductivity for cardiac tissue engineering application, Mater. Sci. Eng., C, 2018, 92, 151160.

18 H. A. A. Hussein, O. M. Darwesh and B. B. Mekki, Environmentally friendly nano-selenium to improve antioxidant system and growth of groundnut cultivars under sandy soil conditions, Biocatal. Agric. Biotechnol, 2019, 18, 1-7.

19 S. Zheng, X. Li, Y. Zhang, Q. Xie, Y. S. Wong, W. Zheng and T. Chen, PEG-nanolized ultrasmall selenium nanoparticles overcome drug resistance in hepatocellular carcinoma HepG2 cells through induction of mitochondria dysfunction, Int. J. Nanomed., 2012, 7, 3939-3949.

20 S. Chaudhary, S. Kumar and S. K. Mehta, Glycol modified gadolinium oxide nanoparticles as a potential template for selective and sensitive detection of 4-nitrophenol, J. Mater. Chem. C, 2015, 3, 8824-8833.

21 S. Chaudhary, P. Sharma, S. Kumar, S. A. Alex, R. Kumar, S. K. Mehta, A. Mukherjee and A. Umar, A comparative multi-assay approach to study the toxicity behaviour of $\mathrm{Eu}_{2} \mathrm{O}_{3}$ nanoparticles, J. Mol. Liq., 2018, 269, 783-795.

22 P. Sharma, S. Kaur, S. Chaudhary, A. Umar and R. Kumar, Bare and nonionic surfactant-functionalized praseodymium oxide nanoparticles: Toxicological studies, Chemosphere, 2018, 209, 1007-1020.

23 S. Chaudhary, P. Sharma, D. Singh, A. Umar and R. Kumar, Chemical and Pathogenic Cleanup of Wastewater Using Surface-Functionalized $\mathrm{CeO}_{2} \quad$ Nanoparticles, ACS Sustainable Chem. Eng., 2017, 5, 6803-6816.

24 K. Jadhav, R. HR, S. Deshpande, S. Jagwani, D. Dhamecha, S. Jalalpure, K. Subburayan and D. Baheti, Phytosynthesis of gold nanoparticles: Characterization, biocompatibility, and evaluation of its osteoinductive potential for application in implant dentistry, Mater. Sci. Eng., C, 2018, 93, 664-670.

25 D. Dhamecha, S. Jalalpure and K. Jadhav, Nepenthes khasiana mediated synthesis of stabilized gold nanoparticles: Characterization and biocompatibility studies, J. Photochem. Photobiol., B, 2016, 154, 108-117.

26 N. Q. Hien, P. D. Tuan, D. Van Phu, L. A. Quoc, N. T. K. Lan, N. N. Duy and T. T. Hoa, Gamma Co-60 ray irradiation synthesis of dextran stabilized selenium nanoparticles and their antioxidant activity, Mater. Chem. Phys., 2018, 205, 29-34.

27 J. M. Zook, R. I. MacCuspie, L. E. Locascio, M. D. Halter and J. T. Elliott, Stable nanoparticle aggregates/agglomerates of different sizes and the effect of their size on hemolytic cytotoxicity, Nanotoxicology, 2011, 5, 517-530.

$28 \mathrm{M}$. Oyaizu, Studies on products of browning reaction. Antioxidative activities of products of browning reaction prepared from glucosamine, J. Nutr. Diet., 1986, 44, 307-315.

29 I. Gulcin, Antioxidant activity of caffeic acid (3,4dihydroxycinnamic acid), Toxicology, 2006, 217, 213-220.

30 H. Liu, X. Qu, H. Tan, J. Song, M. Lei, E. Kim, G. F. Payne and C. Liu, Role of polydopamine's redox-activity on its prooxidant, radical-scavenging, and antimicrobial activities, Acta Biomater., 2019, 88, 181-196.

31 O. Tokumaru, Y. Shuto, K. Ogata, M. Kamibayashi, K. Bacal, H. Takei, I. Yokoi and T. Kitano, Dose-dependency of multiple free radical-scavenging activity of edaravone, $J$. Surg. Res., 2018, 228, 147-153.

32 S. Li, H. Liu, R. Gao, A. Abdurahman, J. Dai and F. Zeng, Aggregation kinetics of microplastics in aquatic 
environment: Complex roles of electrolytes, $\mathrm{pH}$, and natural organic matter, Environ. Pollut., 2018, 237, 126-132.

33 M. Kah, H. Walch and T. Hofmann, Environmental fate of nanopesticides: durability, sorption and photodegradation of nanoformulated clothianidin, Environ. Sci.: Nano, 2018, 5, 882-889.

34 A. A. Markus, J. R. Parsons, E. W. M. Roex, P. de Voogt and R. W. P. M. Laane, Modeling aggregation and sedimentation of nanoparticles in the aquatic environment, Sci. Total Environ., 2015, 506, 323-329.

35 N. Amigo, H. Palza, D. Canales, F. Sepulveda, D. A. Vesco, F. Sepulveda and P. A. Zapata, Effect of starch nanoparticles on the crystallization kinetics and photodegradation of high density polyethylene, Composites, Part B, 2019, 174, 106979-106985.

36 S. K. Mehta, S. Chaudhary, S. Kumar, K. K. Bhasin, K. Torigoe, H. Sakai and M. Abe, Surfactant assisted synthesis and spectroscopic characterization of selenium nanoparticles in ambient conditions, Nanotechnology, 2008, 19, 295601-295612.

37 S. Chaudhary, P. Chauhan, R. Kumar and K. K. Bhasin, Toxicological responses of surfactant functionalized Selenium nanoparticles: A quantitative multi-assay approach, Sci. Total Environ., 2018, 643, 1265-1277. 